TRANSACTIONS OF THE

AMERICAN MATHEMATICAL SOCIETY

Volume 357, Number 2, Pages 443-458

S 0002-9947(04)03655-

Article electronically published on September 2, 2004

\title{
QUANTUM COHOMOLOGY OF PARTIAL FLAG MANIFOLDS
}

\author{
ANDERS SKOVSTED BUCH
}

\begin{abstract}
We give elementary geometric proofs of the structure theorems for the (small) quantum cohomology of partial flag varieties $\mathrm{SL}(n) / P$, including
\end{abstract} the quantum Pieri and quantum Giambelli formulas and the presentation.

\section{INTRODUCTION}

The (small) quantum cohomology ring of a partial flag variety $\mathrm{SL}_{n}(\mathbb{C}) / P$ is a deformation of the usual cohomology ring. The structure constants are the threepoint, genus zero Gromov-Witten invariants, which count the number of rational curves meeting three general Schubert varieties. The remarkable fact that this ring is associative [18, 14 makes it possible to use the associativity relations to compute Gromov-Witten invariants.

The usual approach for understanding this ring consists of proving a presentation for the ring [21, 19, 10, 13, 6, 1, 12], together with a quantum Giambelli formula which expresses the Schubert classes as polynomials in the generators [2, 8, 7]. This information determines the ring as well as all the Gromov-Witten invariants it encodes. In addition, a quantum Pieri formula is known for the multiplication by special Schubert classes [2, 7] 17]. These are the Chern classes of the tautological bundles, and represent the special Schubert varieties defined by a single Schubert condition. Since the special Schubert classes generate the quantum ring, the quantum Pieri formula also determines this ring and its Gromov-Witten invariants.

The purpose of this paper is to give elementary proofs of the above structure theorems for the quantum ring of a partial flag variety. We do this by proving Ciocan-Fontanine's general quantum Pieri formula [7] and by deriving the other results from this formula. The quantum Pieri formula is proved by explicitly solving the underlying Gromov-Witten problem. That is, given three general Schubert varieties, one of which is special, we construct the unique rational curve (of adequate multidegree) meeting these varieties, or prove that none exist. We then rely on Ciocan-Fontanine's proof that the presentation of the quantum ring is a consequence of the quantum Pieri formula, and give another argument that the quantum Giambelli formula is also a consequence.

The original proofs of the quantum formulas relied on intersection theory on hyperquot schemes. In the present paper, these techniques have been replaced with classical Schubert calculus applied to partial flags called the kernel and span of a curve [4, 3]. In particular, if we grant the associativity of quantum cohomology, we make no use of moduli spaces in this paper.

Received by the editors March 12, 2003.

2000 Mathematics Subject Classification. Primary 14N35; Secondary 14M15, $05 \mathrm{E} 15$.

(C)2004 American Mathematical Society 
We also investigate how the theory can best be used to compute Gromov-Witten invariants. To this end, we give algorithms for computing quantum Schubert polynomials and Gromov-Witten invariants. Despite their simplicity, these algorithms in our experience give an efficient method for computing in the quantum ring.

In section 2 we set up notation and recall the structure of the usual cohomology ring of a partial flag variety. We furthermore give the algorithm for computing quantum Schubert polynomials (although it is stated for the usual Schubert polynomials). In section 3 we recall the definition of the quantum ring, state the quantum Pieri formula, and use it to derive the remaining results. We finish this section by explaining the algorithm for computing Gromov-Witten invariants. In section 4 we prove some combinatorial lemmas relating to the the classical and quantum Pieri formulas. Section 5 contains geometric tools for handling curves in partial flag varieties. These combinatorial and geometric tools are finally used to prove the quantum Pieri formula in section 6 .

We thank Sergey Fomin for showing us a very slick proof of Lemma 5, We also thank Ionuţ Ciocan-Fontanine for helpful comments.

\section{Cohomology of Flag VARIEties}

2.1. Cohomology. Set $E=\mathbb{C}^{n}$. Given a strictly increasing sequence of integers $\left(a_{1}<a_{2}<\cdots<a_{k}\right)$ with $a_{1}>0$ and $a_{k}<n$, we let $\mathrm{F} \ell(a ; E)$ be the variety of partial flags $V_{1} \subset V_{2} \subset \cdots \subset V_{k} \subset E$ such that $\operatorname{dim}\left(V_{i}\right)=a_{i}$ for all $i$. For convenience we set $a_{0}=0$ and $a_{k+1}=n$. The dimension of $\mathrm{F} \ell(a ; E)$ is equal to $\sum_{i=1}^{k} a_{i}\left(a_{i+1}-a_{i}\right)$.

Let $S_{n}$ be the group of permutations of $n$ elements. The Schubert varieties in $\mathrm{F} \ell(a ; E)$ are indexed by the set $S_{n} / W_{a}$, where $W_{a} \subset S_{n}$ is the subgroup generated by the simple transpositions $s_{i}=(i, i+1)$ for $i \notin\left\{a_{1}, \ldots, a_{k}\right\}$. Let $S_{n}(a) \subset$ $S_{n}$ denote the set of permutations whose descent positions are contained in the set $\left\{a_{1}, a_{2}, \ldots, a_{k}\right\}$. These permutations are the shortest representatives for the elements in $S_{n} / W_{a}$. Given a fixed full flag $F_{1} \subset F_{2} \subset \cdots \subset F_{n-1} \subset E$ and a permutation $w \in S_{n}(a)$, define the Schubert variety

$$
\Omega_{w}^{(a)}\left(F_{\bullet}\right)=\left\{V_{\bullet} \in \mathrm{F} \ell(a ; E) \mid \operatorname{dim}\left(V_{i} \cap F_{p}\right) \geq \#\left\{t \leq a_{i}: w(t)>n-p\right\} \forall i, p\right\} .
$$

The codimension of this variety is equal to the length $\ell(w)$ of the permutation $w \in S_{n}(a)$.

We let $\Omega_{w}^{(a)}$ denote the fundamental class of $\Omega_{w}^{(a)}\left(F_{\bullet}\right)$ in the cohomology ring $H^{*}(\mathrm{~F} \ell(a ; E))=H^{*}(\mathrm{~F} \ell(a ; E) ; \mathbb{Z})$. The Schubert classes $\Omega_{w}^{(a)}$ form a basis for this ring, for all $w \in S_{n}(a)$. The Schubert class Poincaré dual to $\Omega_{w}^{(a)}$ is the class $\Omega_{w_{0} w w_{a}}^{(a)}$ where $w_{0}=n \ldots 21$ is the longest permutation in $S_{n}$ and $w_{a}$ is the longest permutation in the subgroup $W_{a} \subset S_{n}$, i.e. $w_{a}(j)=a_{i}+a_{i+1}+1-j$ for $a_{i}<j \leq$ $a_{i+1}$.

2.2. Pieri's formula. The Pieri formula gives a rule for multiplying with the Chern classes of the tautological bundles on $\mathrm{F} \ell(a ; E)[15,20]$. Let $t_{i j}$ denote the transposition interchanging $i$ and $j$.

Definition 1. Let $1 \leq r \leq m \leq n-1$ be integers and consider the cyclic permutation $\alpha=s_{r} s_{r+1} \cdots s_{m} \in S_{n}$ of length $\ell=m-r+1$. For permutations $u$ and $w$ we write $u \stackrel{\alpha}{\longrightarrow} w$ if there exist integers $b_{1}, \ldots, b_{\ell}$ and $c_{1}, \ldots, c_{\ell}$ such that 
(1) $b_{i} \leq m<c_{i}$ for all $1 \leq i \leq \ell$;

(2) $w=u t_{b_{1} c_{1}} \ldots t_{b_{\ell} c_{\ell}}$;

(3) $\ell\left(u t_{b_{1} c_{1}} \ldots t_{b_{i} c_{i}}\right)=\ell(u)+i$ for all $1 \leq i \leq \ell$; and

(4) the integers $b_{1}, \ldots, b_{\ell}$ are distinct.

If $m=a_{j}$ for some $j$, then $\alpha$ belongs to $S_{n}(a)$ and corresponds to the special Schubert variety $\Omega_{\alpha}^{(a)}\left(F_{\bullet}\right)$ of points $V_{\bullet} \in \mathrm{F} \ell(a ; E)$ such that $\operatorname{dim}\left(V_{j} \cap F_{n-r}\right) \geq \ell$. Its Schubert class is given by $\Omega_{\alpha}^{(a)}=(-1)^{\ell} c_{\ell}\left(\mathcal{V}_{j}\right) \in H^{*}(\mathrm{~F} \ell(a ; E))$, where $\mathcal{V}_{1} \subset \cdots \subset$ $\mathcal{V}_{k} \subset E$ denotes the tautological flag on $\mathrm{F} \ell(a ; E)$. The Pieri formula states that for any permutation $u \in S_{n}(a)$ we have

$$
\Omega_{\alpha}^{(a)} \cdot \Omega_{u}^{(a)}=\sum_{u \stackrel{\alpha}{\rightarrow} w} \Omega_{w}^{(a)} .
$$

2.3. Presentation. We let $\mathrm{F} \ell(E)=\mathrm{F} \ell(1,2, \ldots, n-1 ; E)$ denote the full flag variety of $E$, and we denote its Schubert varieties and Schubert classes by $\Omega_{w}\left(F_{\bullet}\right)$ and $\Omega_{w}$, respectively, for $w \in S_{n}$. The cohomology ring of $\mathrm{F} \ell(E)$ has the presentation

$$
H^{*}(\mathrm{~F} \ell(E))=\mathbb{Z}\left[x_{1}, \ldots, x_{n}\right] /\left(e_{1}^{n}, \ldots, e_{n}^{n}\right)
$$

where $e_{i}^{n}=e_{i}\left(x_{1}, \ldots, x_{n}\right)$ is the $i$ th elementary symmetric polynomial in $n$ variables. This presentation maps $x_{i}$ to the class $\Omega_{s_{i}}-\Omega_{s_{i-1}}$, which is identical to the Chern class $-c_{1}\left(\mathcal{V}_{i} / \mathcal{V}_{i-1}\right)$.

In this presentation the Schubert class $\Omega_{w}$ is represented by the Schubert polynomial $\mathfrak{S}_{w}=\mathfrak{S}_{w}\left(x_{1}, \ldots, x_{n-1}\right)$ of Lascoux and Schützenberger [15. It is defined as follows. If $w=w_{0}$ is the longest permutation in $S_{n}$, then we set

$$
\mathfrak{S}_{w_{0}}=x_{1}^{n-1} x_{2}^{n-2} \cdots x_{n-1} .
$$

Otherwise we can find a simple transposition $s_{i} \in S_{n}$ such that $\ell\left(w s_{i}\right)=\ell(w)+1$. In this case we define

$$
\mathfrak{S}_{w}=\frac{\mathfrak{S}_{w s_{i}}\left(x_{1}, \ldots, x_{i}, x_{i+1}, \ldots, x_{n}\right)-\mathfrak{S}_{w s_{i}}\left(x_{1}, \ldots, x_{i+1}, x_{i}, \ldots, x_{n}\right)}{x_{i}-x_{i+1}} .
$$

An important property of these polynomials is that they multiply with the same structure constants as those of the Schubert classes they represent. In particular, the Pieri formula (1) also holds as an identity of Schubert polynomials.

The ring $H^{*}(\mathrm{~F} \ell(a ; E))$ is isomorphic to the subring of $\mathbb{Z}\left[x_{1}, \ldots, x_{n}\right] /\left(e_{1}^{n}, \ldots, e_{n}^{n}\right)$ generated by the elementary symmetric polynomials $y_{i}^{p}=e_{i}\left(x_{a_{p-1}+1}, \ldots, x_{a_{p}}\right)$ for $1 \leq p \leq k+1$ and $1 \leq i \leq a_{p}-a_{p-1}$. Notice that $e_{j}^{n}=\sum y_{i_{1}}^{1} \ldots y_{i_{k+1}}^{k+1}$ where the sum is over all sequences $\left(i_{1}, \ldots, i_{k+1}\right)$ such that $0 \leq i_{p} \leq a_{p}-a_{p-1}$ and $\sum i_{p}=j$. We therefore get the direct presentation

$$
H^{*}(\mathrm{~F} \ell(a ; E))=\mathbb{Z}\left[y_{1}^{1}, \ldots, y_{a_{1}}^{1}, y_{1}^{2}, \ldots, y_{a_{2}-a_{1}}^{2}, \ldots, y_{1}^{k+1}, \ldots, y_{n-a_{k}}^{k+1}\right] /\left(e_{1}^{n}, \ldots, e_{n}^{n}\right),
$$

which maps $y_{i}^{p}$ to $(-1)^{i} c_{i}\left(\mathcal{V}_{p} / \mathcal{V}_{p-1}\right)=c_{i}\left(\mathcal{Q}_{p}\right)$, where $\mathcal{Q}_{p}$ is the dual of the bundle $\mathcal{V}_{p} / \mathcal{V}_{p-1}$

2.4. An algorithm for Schubert polynomials. If $w \in S_{n}(a)$, then the Schubert polynomial $\mathfrak{S}_{w}$ is symmetric in each interval of variables $x_{a_{p-1}+1}, \ldots, x_{a_{p}}$, so $\mathfrak{S}_{w}$ can be written as a polynomial in the variables $y_{i}^{p}$. This gives a representative of the Schubert class $\Omega_{w}^{(a)}$ in the above presentation for $H^{*}(\mathrm{~F} \ell(a ; E))$. 
We will here give a simple method for expressing a Schubert polynomial $\mathfrak{S}_{w}$ for $w \in S_{n}(a)$ as an integral linear combination

$$
\mathfrak{S}_{w}=\sum c_{i_{a_{1}}, \ldots, i_{n-1}} e_{i_{a_{1}}, \ldots, i_{n-1}}^{(a)}
$$

of products of the form

$$
e_{i_{a_{1}}, \ldots, i_{n-1}}^{(a)}=\prod_{p=1}^{k} \prod_{r=a_{p}}^{a_{p+1}-1} e_{i_{r}}\left(x_{1}, \ldots, x_{a_{p}}\right)
$$

for sequences $\left(i_{a_{1}}, \ldots, i_{n-1}\right)$ such that for $a_{p} \leq r<a_{p+1}$ we have $0 \leq i_{r} \leq a_{p}$. In fact, if we demand that $i_{a_{p}} \leq i_{a_{p}+1} \leq \cdots \leq i_{a_{p+1}-1}$ for all $p$, then the polynomials $e_{i_{a_{1}}, \ldots, i_{n-1}}^{(a)}$ are linearly independent, so the obtained coefficients $c_{i_{a_{1}}, \ldots, i_{n-1}}$ are uniquely determined integers.

Schubert polynomials in the form (2) were used by Fomin, Gelfand, and Postnikov 8 and by Ciocan-Fontanine [7] to define quantum Schubert polynomials. This application will be explained in 3.4. Notice that the expression (2) may easily be converted to an expression for $\mathfrak{S}_{w}$ in the $y_{i}^{p}$-variables, thus giving the representative of the class $\Omega_{w}^{(a)}$ in the presentation for $H^{*}(\mathrm{~F} \ell(a ; E))$.

The polynomial $\mathfrak{S}_{w}$ can be expressed in the form (2) as follows. Choose $p \leq k$ maximal such that $w\left(a_{p}+1\right) \neq a_{p}+1$, and define $u \in S_{n}(a)$ by

$$
u(i)= \begin{cases}w(i) & \text { if } i \leq a_{p} \text { and } w(i)<w\left(a_{p}+1\right), \\ w(i)-1 & \text { if } i \leq a_{p} \text { and } w(i)>w\left(a_{p}+1\right), \\ w(i+1)-1 & \text { if } i>a_{p} .\end{cases}
$$

Set $\alpha=s_{w\left(a_{p}+1\right)} \cdots s_{a_{p}-1} s_{a_{p}} \in S_{n}(a)$. Then we have $\mathfrak{S}_{\alpha}=e_{a_{p}+1-w\left(a_{p}+1\right)}^{a_{p}}$. We claim that the identity

$$
\mathfrak{S}_{w}=\mathfrak{S}_{u} \cdot e_{a_{p}+1-w\left(a_{p}+1\right)}^{a_{p}}-\sum_{u \stackrel{\alpha}{\rightarrow} v \neq w} \mathfrak{S}_{v}
$$

can be used recursively to obtain the required expansion of $\mathfrak{S}_{w}$.

Notice that since $w \in S_{n}(a)$ we automatically have $w\left(a_{p}+1\right)<a_{p}+1$. The identity (3) is true by the Pieri formula because $u \stackrel{\alpha}{\rightarrow} w$. We must show that the recursive process terminates and that the resulting expression for $\mathfrak{S}_{w}$ has the required form (2).

For $r<n$ we let $S_{r} \subset S_{n}$ denote the subgroup of permutations fixing the set $\{r+1, \ldots, n\}$. Choose $r$ minimal such that $w \in S_{r}$. Then $r \leq a_{p+1}$ and $u \in S_{r-1}$. Suppose $\mathfrak{S}_{v}$ occurs in the product $\mathfrak{S}_{u} \cdot \mathfrak{S}_{\alpha}$. Then Lemma 2 of section 4 , with $m=a_{p}$, implies that $w(i) \leq v(i) \leq u(i)$ for $i \geq a_{p}+2$. Now it is immediate from Definition 1 that $\sum_{i=a_{p}+1}^{n}(u(i)-v(i)) \geq \ell(\alpha)$. Since $\ell(\alpha)=\sum_{i=a_{p}+1}^{n}(u(i)-w(i))$, we conclude that $v\left(a_{p}+1\right) \leq w\left(a_{p}+1\right)$, and if equality holds then $v=w$. Since we also have $v \in S_{r}$, this immediately implies termination. The resulting expression for $\mathfrak{S}_{w}$ is of the form (2) by induction on $r$.

Example 1. For $n=7$ and $a=(2,4)$ we get

$$
\mathfrak{S}_{1536247}=\mathfrak{S}_{1425367} \cdot e_{3}^{4}-\mathfrak{S}_{2436157}=\left(e_{1}^{2} e_{2}^{4}-e_{3}^{4}\right) e_{3}^{4}-e_{1}^{2} e_{1}^{4} e_{4}^{4} .
$$

When using equation (3) in real life, it is essential to remember the Schubert polynomials which have already been calculated in the recursive process, since otherwise the calculation of such polynomials can be repeated multiple times. However, 
when this precaution is taken, the algorithm performs well. For alternative formulas for Schubert polynomials with relevance to partial flag varieties we refer to [5].

\section{QuANTUM COHOMOLOGY OF FLAG VARIETIES}

3.1. Gromov-Witten invariants. A rational curve in $\mathrm{F} \ell(a ; E)$ is the image of a regular map $\mathbb{P}^{1} \rightarrow \mathrm{F} \ell(a ; E)$. (We will tolerate that a rational curve can be a point according to this definition.) The multidegree of a curve $C \subset \mathrm{F} \ell(a ; E)$ is the sequence $d=\left(d_{1}, \ldots, d_{k}\right)$ where $d_{i}$ is the number of points in the intersection $C \cap \Omega_{s_{a_{i}}}^{(a)}\left(F_{\bullet}\right)$ for any general flag $F_{\text {. of }} E$. Thus, if $C$ is not a point, then the cohomology class of $C$ is equal to $\sum_{i=1}^{k} d_{i} \Omega_{w_{0} s_{a_{i}} w_{a}}^{(a)}$.

Given $u, v, w \in S_{n}(a)$ and a multidegree $d$ such that $\ell(u)+\ell(v)+\ell(w)=$ $\operatorname{dim} \mathrm{F} \ell(a ; E)+\sum_{i=1}^{k} d_{i}\left(a_{i+1}-a_{i-1}\right)$, the (three-point, genus zero) Gromov-Witten invariant $\left\langle\Omega_{u}^{(a)}, \Omega_{v}^{(a)}, \Omega_{w}^{(a)}\right\rangle_{d}$ is defined to be the number of rational curves in $\mathrm{F} \ell(a ; E)$ of multidegree $d$ meeting all of the Schubert varieties $\Omega_{u}^{(a)}\left(F_{\bullet}\right), \Omega_{v}^{(a)}\left(G_{\bullet}\right)$, and $\Omega_{w}^{(a)}\left(H_{\bullet}\right)$ for fixed flags $F_{\bullet}, G_{\bullet}, H_{\bullet}$ in general position. When $\ell(u)+\ell(v)+\ell(w) \neq$ $\operatorname{dim} \mathrm{F} \ell(a ; E)+\sum d_{i}\left(a_{i+1}-a_{i-1}\right)$ we set $\left\langle\Omega_{u}^{(a)}, \Omega_{v}^{(a)}, \Omega_{w}^{(a)}\right\rangle_{d}=0$.

Let $q_{1}, \ldots, q_{k}$ be independent variables and write $\mathbb{Z}[q]=\mathbb{Z}\left[q_{1}, \ldots, q_{k}\right]$. The (small) quantum cohomology ring of $\mathrm{F} \ell(a ; E)$ is a $\mathbb{Z}[q]$-algebra, which as a $\mathbb{Z}[q]$ module is free with a basis of quantum Schubert classes $\sigma_{w}^{(a)}$ :

$$
Q H^{*}(\mathrm{~F} \ell(a ; E))=\bigoplus_{w \in S_{n}(a)} \mathbb{Z}[q] \sigma_{w}^{(a)} .
$$

Multiplication is defined by the formula

$$
\sigma_{u}^{(a)} \cdot \sigma_{v}^{(a)}=\sum_{w, d}\left\langle\Omega_{u}^{(a)}, \Omega_{v}^{(a)}, \Omega_{w_{0} w w_{a}}^{(a)}\right\rangle_{d} q^{d} \sigma_{w}^{(a)}
$$

where the sum is over all $w \in S_{n}(a)$ and multidegrees $d$, and $q^{d}=q_{1}^{d_{1}} q_{2}^{d_{2}} \cdots q_{k}^{d_{k}}$.

It is a non-trivial fact that this product is associative [18, 14, 9]. The ring $Q H^{*}(\mathrm{~F} \ell(a ; E))$ has a natural grading, where the degree of $\sigma_{w}^{(a)}$ is the length $\ell(w)$, while each variable $q_{i}$ has degree $a_{i+1}-a_{i-1}$. If we set $q_{i}=0$ for each $i$, we recover the usual cohomology ring $H^{*}(\mathrm{~F} \ell(a ; E))$.

3.2. The quantum Pieri formula. The central result about the structure of the quantum ring $Q H^{*}(\mathrm{~F} \ell(a ; E))$ is the quantum Pieri formula of Ciocan-Fontanine [7]. This result generalizes the quantum Pieri formula for Grassmannians 2 and the quantum Monk's formula for full flag varieties [8]. In the case of full flag varieties, Postnikov has given an equivalent but simpler statement of the quantum Pieri formula, as well as a combinatorial proof based on the quantum Monk's formula [17. We will give an elementary geometric proof of Ciocan-Fontanine's result in the last section.

We will call a sequence $d=\left(d_{1}, \ldots, d_{k}\right)$ of non-negative integers a Pieri sequence with maximum at position $j$, if $\left(d_{1}, \ldots, d_{j}\right)$ is weakly increasing, $\left(d_{j}, \ldots, d_{k}\right)$ is weakly decreasing, and if we set $d_{0}=d_{k+1}=0$ then $\left|d_{i}-d_{i+1}\right| \leq 1$ for $0 \leq i \leq k$. Given such a sequence, we set $\gamma_{d}=\tau_{1} \tau_{2} \cdots \tau_{k} \in S_{n}$ where $\tau_{i}$ is the permutation which interchanges the intervals $\left[a_{i}-d_{i}+1, a_{i}\right]$ and $\left[a_{i}+1, a_{i+1}\right]$. In other words, 
$\tau_{i}$ is defined by

$$
\tau_{i}(p)= \begin{cases}p+a_{i+1}-a_{i} & \text { if } a_{i}-d_{i}<p \leq a_{i} \\ p-d_{i} & \text { if } a_{i}<p \leq a_{i+1} \\ p & \text { otherwise }\end{cases}
$$

Theorem 1 (Quantum Pieri formula [7]). Let $\alpha=s_{r} s_{r+1} \cdots s_{a_{j}}$ and $u \in S_{n}(a)$ be permutations. Then

$$
\sigma_{\alpha}^{(a)} \cdot \sigma_{u}^{(a)}=\sum q^{d} \sigma_{w}^{(a)}
$$

where the sum is over all Pieri sequences $d$ with maximum at position $j$ and permutations $w \in S_{n}\left(\right.$ a) such that (i) $\ell\left(u \gamma_{d}\right)=\ell(u)-\ell\left(\gamma_{d}\right)$; (ii) $\ell\left(w w_{a} \gamma_{d}\right)=$ $\ell\left(w w_{a}\right)+\ell\left(\gamma_{d}\right)$; and (iii) $u \gamma_{d} \stackrel{\bar{\alpha}}{\rightarrow} w w_{a} \gamma_{d} w_{b}$ where $b=a-d=\left(a_{1}-d_{1}, \ldots, a_{k}-d_{k}\right)$ and $\bar{\alpha}=s_{r} s_{r+1} \cdots s_{b_{j}}$.

An equivalent symmetric version of this theorem is given in section [6] Notice that condition (iii) implicitly implies that $d_{j} \leq \ell(\alpha)$.

Given a Pieri sequence $d$ with maximum at position $j$, set $h_{p}=\min \left\{i: d_{i}=p\right\}$ and $l_{p}=\max \left\{i: d_{i}=p\right\}$ for each $1 \leq p \leq d_{j}$. With this notation we have $\gamma_{d}\left(b_{l_{p}+1}\right)=a_{h_{p}}$, while $\gamma_{d}(i)=i+p$ if $b_{h_{p}}<i \leq b_{h_{p+1}}$ or if $b_{l_{p+1}+1}<i<b_{l_{p}+1}$. It follows that $\ell\left(u \gamma_{d}\right)=\ell(u)-\ell\left(\gamma_{d}\right)$ if and only if $u\left(a_{h_{p}}\right)>u(i)$ for all $p$ and $a_{h_{p}}<i \leq a_{l_{p}+1}$ (cf. 7, Remark 3.2 (ii)]).

Example 2. Let $\alpha=s_{2} s_{3} s_{4}$ and $u=3715246$. We will compute the product $\sigma_{\alpha}^{(a)} \cdot \sigma_{u}^{(a)}$ in the ring $Q H^{*} \mathrm{~F} \ell\left(2,4 ; \mathbb{C}^{7}\right)$. First observe that the Pieri sequences $d$ with maximum at position 2 such that $\ell\left(u \gamma_{d}\right)=\ell(u)-\ell\left(\gamma_{d}\right)$ are $(0,0)$ and $(1,1)$. The first of these contributes $\sum_{u} \stackrel{\alpha}{\longrightarrow} \sigma_{w}^{(a)}=\sigma_{4726135}^{(a)}$. For $d=(1,1)$ we have $\bar{\alpha}=s_{2} s_{3}$, and $u \gamma_{d}=3152467 \stackrel{\bar{\alpha}}{\rightarrow} v$ when $v$ is one of the permutations 4251367, 3261457, or 4162357. The first two of these satisfy $\ell\left(v w_{b} \gamma_{d}^{-1}\right)=\ell\left(v w_{b}\right)-\ell\left(\gamma_{d}\right)$, and they contribute $q_{1} q_{2} \sigma_{1425367}^{(a)}+q_{1} q_{2} \sigma_{1326457}^{(a)}$. In conclusion we have

$$
\sigma_{\alpha}^{(a)} \cdot \sigma_{u}^{(a)}=\sigma_{4726135}^{(a)}+q_{1} q_{2} \sigma_{1425367}^{(a)}+q_{1} q_{2} \sigma_{1326457}^{(a)} .
$$

3.3. Structure of the quantum ring. The presentation of $Q H^{*} \mathrm{~F} \ell(a ; E)$ is due to Astashkevich and Sado [1] and Kim [11, 12] (see also [21, 19] for the Grassmannian case, and [10, 13 6] for the case of full flag varieties). In this section we sketch how to recover this presentation from the quantum Pieri formula. We follow CiocanFontanine's paper [7].

Let $\phi: H^{*}(\mathrm{~F} \ell(a ; E)) \rightarrow Q H^{*}(\mathrm{~F} \ell(a ; E))$ be the linear map which sends each Schubert class $\Omega_{w}^{(a)}$ to the corresponding quantum Schubert class $\sigma_{w}^{(a)}$. The presentation of $Q H^{*}(\mathrm{~F} \ell(a ; E))$ uses variables $y_{i}^{j}$ and $q_{j}$, and maps $y_{i}^{j}$ to $(-1)^{i} \phi\left(c_{i}\left(\mathcal{V}_{j} / \mathcal{V}_{j-1}\right)\right)=$ $\phi\left(c_{i}\left(\mathcal{Q}_{j}\right)\right)$.

Set $\alpha_{i, j}=s_{a_{j}-i+1} s_{a_{j}-i+2} \cdots s_{a_{j}}$ and $\beta_{i, j}=s_{a_{j}+i-1} s_{a_{j}+i-2} \cdots s_{a_{j}} \in S_{n}(a)$. Then $\mathfrak{S}_{\alpha_{i, j}}=e_{i}\left(x_{1}, \ldots, x_{a_{j}}\right)$ is the elementary symmetric polynomial and $\mathfrak{S}_{\beta_{i, j}}=$ $h_{i}\left(x_{1}, \ldots, x_{a_{j}}\right)$ is the complete symmetric polynomial in $a_{j}$ variables. Using the Pieri formula (11), it follows that for $i \leq a_{j}-a_{j-1}$ we have (cf. [7] Lemma 3.5])

$$
c_{i}\left(\mathcal{Q}_{j}\right)=\sum_{p=0}^{i}(-1)^{p} \Omega_{\alpha_{i-p, j}}^{(a)} \cdot \Omega_{\beta_{p, j-1}}^{(a)}=\sum_{p=0}^{i}(-1)^{p} \Omega_{\beta_{p, j-1} \alpha_{i-p, j}}^{(a)} .
$$


We therefore get

$$
\phi\left(c_{i}\left(\mathcal{Q}_{j}\right)\right)=\sum_{p=0}^{i}(-1)^{p} \sigma_{\beta_{p, j-1} \alpha_{i-p, j}}^{(a)} .
$$

Define quantum elementary symmetric polynomials $E_{i}^{j}$ as follows. If $i=j=0$, then set $E_{0}^{0}=1$. If $j<0$ or $i<0$ or $i>a_{j}$, then set $E_{i}^{j}=0$. Otherwise, if $0 \leq i \leq a_{j} \neq 0$, then define inductively

$$
E_{i}^{j}=E_{i}^{j-1}+\sum_{r=1}^{a_{j}-a_{j-1}} y_{r}^{j} E_{i-r}^{j-1}-(-1)^{a_{j}-a_{j-1}} q_{j-1} E_{i-a_{j}+a_{j-2}}^{j-2} .
$$

For example, if $n=7$ and $a=(2,4)$, then we get

$$
\begin{aligned}
E_{5}^{3} & =E_{5}^{2}+y_{1}^{3} E_{4}^{2}+y_{2}^{3} E_{3}^{2}+y_{3}^{3} E_{2}^{2}-(-1)^{3} q_{2} E_{0}^{1} \\
& =0+y_{1}^{3}\left(y_{2}^{2} y_{2}^{1}-q_{1}\right)+y_{2}^{3}\left(y_{1}^{2} y_{2}^{1}+y_{2}^{2} y_{1}^{1}\right)+y_{3}^{3}\left(y_{2}^{1}+y_{1}^{2} y_{1}^{1}+y_{2}^{2}\right)+q_{2} .
\end{aligned}
$$

We claim that the quantum ring has the presentation

$$
Q H^{*}(\mathrm{~F} \ell(a ; E))=\mathbb{Z}[y, q] /\left(E_{1}^{k+1}, \ldots, E_{n}^{k+1}\right)
$$

where each variable $y_{i}^{j}$ is mapped to $\phi\left(c_{i}\left(\mathcal{Q}_{j}\right)\right)$ for $1 \leq j \leq k+1$ and $1 \leq i \leq$ $a_{j}-a_{j-1}$.

More generally, if we replace each $y_{i}^{j}$ with $\phi\left(c_{i}\left(\mathcal{Q}_{j}\right)\right)$, then $E_{i}^{j}$ maps to $\sigma_{\alpha_{i, j}}^{(a)}$ for $j \leq k$ while $E_{i}^{k+1}$ becomes zero. In fact, since a symmetric functions calculation shows that this is true in cohomology after setting $q_{j}=0$ for all $j$, we only need to determine the $q$-terms which arise when the sum $\sum_{r=1}^{a_{j}-a_{j-1}} \phi\left(c_{r}\left(\mathcal{Q}_{j}\right)\right) \cdot \sigma_{\alpha_{i-r, j-1}}^{(a)}$ is expanded. Here one observes that, if $d$ is a non-zero Pieri sequence with maximum at position $j-1$ and if $\ell\left(\beta_{p, j-1} \alpha_{r-p, j} \gamma_{d}\right)=\ell\left(\beta_{p, j-1} \alpha_{r-p, j}\right)-\ell\left(\gamma_{d}\right)$, then $r=$ $p=a_{j}-a_{j-1}$ and $d=(0, \ldots, 0,1,0, \ldots, 0)$ has a single one at position $j-1$. Furthermore, when $r=a_{j}-a_{j-1}$ the product $\sigma_{\beta_{r, j-1}}^{(a)} \cdot \sigma_{\alpha_{i-r, j-1}}^{(a)}$ contains no $q$ terms for $i<a_{j}-a_{j-2}$, while it has exactly one when $i \geq a_{j}-a_{j-2}$, namely $q_{j-1} \sigma_{\alpha_{i-a_{j}+a_{j-2}, j-2}}^{(a)}$. For more details we refer to [7, Lemma 3.6].

3.4. The quantum Giambelli formula. For a sequence $\left(i_{a_{1}}, \ldots, i_{n-1}\right)$ such that $0 \leq i_{r} \leq a_{j}$ for each $a_{j} \leq r<a_{j+1}$, set

$$
E_{i_{a_{1}}, \ldots, i_{n-1}}^{(a)}=\prod_{j=1}^{k} \prod_{r=a_{j}}^{a_{j+1}-1} E_{i_{r}}^{j} .
$$

Ciocan-Fontanine has given a geometric proof that no $q$-terms occur in the expansion of the corresponding product of quantum Schubert classes [7, Thm. 3.14]. In other words, $\phi$ maps the cohomology class represented by $e_{i_{a_{1}}, \ldots, i_{n-1}}^{(a)}$ to the quantum class given by $E_{i_{a_{1}}, \ldots, i_{n-1}}^{(a)}$. We will here deduce this fact from Theorem 1 .

Suppose $u \in S_{n}(a) \cap S_{r}$ for some $r<a_{j+1}$. Then $\sigma_{u}^{(a)} \cdot \sigma_{\alpha_{i, j}}^{(a)}=\phi\left(\Omega_{u}^{(a)} \cdot \Omega_{\alpha_{i, j}}^{(a)}\right)$, which follows because $\ell\left(u \gamma_{d}\right)>\ell(u)-\ell\left(\gamma_{d}\right)$ for all non-zero Pieri sequences $d$ with maximum at position $j$. Lemma 2 of section 4 furthermore implies that all terms $\Omega_{w}^{(a)}$ in the product $\Omega_{u}^{(a)} \cdot \Omega_{\alpha_{i, j}}^{(a)}$ satisfy $w \in S_{n}(a) \cap S_{r+1}$. For each $a_{1} \leq r \leq n-1$ we 
set $\alpha(r)=\alpha_{i_{r}, j}$ where $j$ is maximal such that $a_{j} \leq r$. By induction on $r$, the above comments imply that $\sigma_{\alpha\left(a_{1}\right)}^{(a)} \cdot \sigma_{\alpha\left(a_{1}+1\right)}^{(a)} \cdots \sigma_{\alpha(r)}^{(a)}=\phi\left(\Omega_{\alpha\left(a_{1}\right)}^{(a)} \cdot \Omega_{\alpha\left(a_{1}+1\right)}^{(a)} \cdots \Omega_{\alpha(r)}^{(a)}\right)$ and that all terms $\sigma_{w}^{(a)}$ in this product satisfy $w \in S_{r+1}$. In particular, the class given by $E_{i_{a_{1}}, \ldots, i_{n-1}}^{(a)}$ contains no $q$-terms.

Define the partial quantum Schubert polynomial for a permutation $w \in S_{n}(a)$ by

$$
\mathfrak{S}_{w}^{q}=\sum c_{i_{a_{1}}, \ldots, i_{n-1}} E_{i_{a_{1}}, \ldots, i_{n-1}}^{(a)}
$$

The coefficients $c_{i_{a_{1}}, \ldots, i_{n-1}}$ are defined by (2). In particular, this definition depends on the sequence $a$.

By applying $\phi$ to the classes represented by either side of (2), it follows that $\mathfrak{S}_{w}^{q}$ is a representative for the quantum Schubert class $\sigma_{w}^{(a)}$ in the presentation of $Q H^{*}(\mathrm{~F} \ell(a ; E))$. In other words, $\mathfrak{S}_{w}^{q}$ is a quantum Giambelli formula. This result is due to Bertram 2 for Grassmannians, to Fomin, Gelfand, and Postnikov for full flag varieties [8], and to Ciocan-Fontanine in general [7].

Notice that the identity (3) gives the direct recursive formula

$$
\mathfrak{S}_{w}^{q}=\mathfrak{S}_{u}^{q} \cdot E_{a_{p}+1-w\left(a_{p}+1\right)}^{p}-\sum_{u \stackrel{\alpha}{\longrightarrow} v \neq w} \mathfrak{S}_{v}^{q}
$$

where $p, u$, and $\alpha$ are chosen as in 2.4. For full flag varieties one can alternatively use a quantum version of the transition formula, which is based on the quantum Monk's formula (see [8, §8] and [16, (4.16)]).

3.5. Computing Gromov-Witten invariants. By definition of the quantum product (4), a Gromov-Witten invariant $\left\langle\Omega_{u}^{(a)}, \Omega_{v}^{(a)}, \Omega_{w}^{(a)}\right\rangle_{d}$ on $\mathrm{F} \ell(a ; E)$ can be computed by extracting the coefficient of $q^{d} \sigma_{w_{0}}^{(a)} w w_{a}$ in the expansion of the product $\sigma_{u}^{(a)} \cdot \sigma_{v}^{(a)} \in Q H^{*}(\mathrm{~F} \ell(a ; E))$.

The following method may be used to compute this product. Start by expressing the quantum class $\sigma_{u}^{(a)}$ as a polynomial in the classes $\sigma_{\alpha_{i j}}^{(a)}$. This can be done using equation (5). Then let this polynomial act on the class $\sigma_{v}^{(a)}$ using the quantum Pieri formula (Thm. 1). The result is the desired expansion.

Practical experiments indicate that this method is quite efficient. For example, it vastly outperforms the Gröbner basis methods suggested in [8]. Notice that the roles of $u, v$, and $w$ can be permuted. Often (but not always) the best choice is to let the quantum Schubert polynomial for the shortest permutation act on one of the other quantum Schubert classes. Notice also that this method for computing GromovWitten invariants does not make any use of the presentation of the quantum ring.

Example 3. We will compute the number of rational curves in $\mathrm{F} \ell\left(2,4 ; \mathbb{C}^{7}\right)$ of multidegree $(2,3)$, which pass through two general points and meet the Schubert variety $\Omega_{3417256}^{(a)}\left(F_{\bullet}\right)$. In other words, we compute the Gromov-Witten invariant $\left\langle\Omega_{6745123}^{(a)}, \Omega_{6745123}^{(a)}, \Omega_{3417256}^{(a)}\right\rangle_{(2,3)}$. Using equation (5), we obtain the point class as $\sigma_{6745123}^{(a)}=\left(\sigma_{\alpha_{2,1}}^{(a)}\right)^{2}\left(\sigma_{\alpha_{4,2}}^{(a)}\right)^{3}$. Now using the quantum Pieri formula repeatedly, we 
obtain

$$
\begin{aligned}
\sigma_{6745123}^{(a)} \cdot \sigma_{3417256}^{(a)} & =\left(\sigma_{\alpha_{2}, 1}^{(a)}\right)^{2}\left(\sigma_{\alpha_{4,2}}^{(a)}\right)^{3} \cdot \sigma_{3417256}^{(a)} \\
& =\left(\sigma_{\alpha_{2}, 1}^{(a)}\right)^{2}\left(\sigma_{\alpha_{4,2}}^{(a)}\right)^{2} \cdot q_{2} \sigma_{4512367}^{(a)} \\
& =\left(\sigma_{\alpha_{2}, 1}^{(a)}\right)^{2} \sigma_{\alpha_{4,2}}^{(a)} \cdot q_{2} \sigma_{5623147}^{(a)} \\
& =\left(\sigma_{\alpha_{2}, 1}^{(a)}\right)^{2} \cdot q_{2} \sigma_{6734125}^{(a)} \\
& =\sigma_{\alpha_{2,1}}^{(a)} \cdot\left(q_{1} q_{2} \sigma_{3746125}^{(a)}+q_{1} q_{2}^{2} \sigma_{1734256}^{(a)}\right) \\
& =q_{1}^{2} q_{2} \sigma_{3467125}^{(a)}+q_{1}^{2} q_{2}^{2} \sigma_{1436257}^{(a)}+q_{1}^{2} q_{2}^{2} \sigma_{1347256}^{(a)}+q_{1}^{2} q_{2}^{3} \sigma_{1234567}^{(a)} .
\end{aligned}
$$

The Gromov-Witten invariant of interest is the coefficient to $q_{1}^{2} q_{2}^{3} \sigma_{1234567}^{(a)}$ in this product, so it is equal to one.

\section{Combinatorics of the Pieri Rule}

In this section we prove some lemmas concerning the Pieri and quantum Pieri formulas. As in $\$ 2.2$ we set $\alpha=s_{r} s_{r+1} \cdots s_{m} \in S_{n}$ and $\ell=\ell(\alpha)$.

Lemma 1. Let $u \stackrel{\alpha}{\longrightarrow} w$ and let $b$ and $c$ be sequences satisfying Definition 1 Suppose $c_{i} \neq c_{i+1}$. Then we can interchange the $i$-th and $(i+1)$-th indices in $b$ and $c$, i.e., the sequences $b^{\prime}=\left(b_{1}, \ldots, b_{i+1}, b_{i}, \ldots, b_{\ell}\right)$ and $c^{\prime}=\left(c_{1}, \ldots, c_{i+1}, c_{i}, \ldots, c_{\ell}\right)$ also satisfy Definition 1 .

Proof. The sequences $b^{\prime}$ and $c^{\prime}$ clearly satisfy properties (1) and (4). Conditions (2) and (3) hold because the transposition $t_{b_{i} c_{i}}$ commutes with $t_{b_{i+1} c_{i+1}}$.

The following fact has already been used in $\$ 2.4$ and $\$ 3.3$

Lemma 2. Let $u \stackrel{\alpha}{\rightarrow} w$ and suppose $u$ has no descents after position $m$. Then for all $j \geq m+2$ we have $u(j-1)<w(j) \leq u(j)$.

Proof. Let $b$ and $c$ be sequences satisfying Definition 1 By Lemma 1 we may assume that $c_{1}=\cdots=c_{p}=j$ and $c_{i} \neq j$ for $i>p$. It then follows from property (3) and induction on $i$ that each permutation $u t_{b_{1} c_{1}} \cdots t_{b_{i} c_{i}}$ maps $j$ to a value greater than $u(j-1)$.

Lemma 3. Let $u \stackrel{\alpha}{\longrightarrow} w$ and suppose that for some $l>m$ we have $u(i)<u(l)$ for all $m<i<l$. Then for all $j \leq m$ such that $u(j)<u(l)$ we have $w(j) \leq u(l)$.

Proof. Let $b$ and $c$ be sequences satisfying Definition 1. We may assume that $w(j) \neq u(j)$, so $j=b_{p}$ for some $p$. By Lemma 1 we may furthermore assume that $c_{1}=c_{2}=\cdots=c_{p}$.

Set $u^{\prime}=u t_{b_{1} c_{1}} \cdots t_{b_{p-1} c_{p-1}}$. If $c_{p} \leq l$, then $w(j)=u^{\prime}\left(c_{p}\right) \leq u\left(c_{p}\right) \leq u(l)$. On the other hand, if $c_{p}>l$, then since $u^{\prime}(j)<u^{\prime}(l)$ and $\ell\left(u^{\prime} t_{b_{p} c_{p}}\right)=\ell\left(u^{\prime}\right)+1$, we must have $u^{\prime}\left(c_{p}\right)<u^{\prime}(l)$, so once again we get

$$
w(j)=u^{\prime}\left(c_{p}\right)<u^{\prime}(l)=u(l),
$$

as required.

If $x_{1}, \ldots, x_{p}$ are elements of a vector space $E$, we let $\left\langle x_{1}, \ldots, x_{p}\right\rangle \subset E$ denote the linear span of these vectors. 
Lemma 4. Let $\left\{e_{1}, \ldots, e_{n}\right\}$ be a basis for a vector space $E$ and let $u, w \in S_{n}$ be permutations such that $u \stackrel{\alpha}{\longrightarrow} w$. Suppose $x_{1}, \ldots, x_{n} \in E$ are elements satisfying the following conditions:

(i) If $i \leq m$ and $u(i)=w(i)$, then $x_{i}=e_{u(i)}$.

(ii) If $i \leq m$ and $u(i) \neq w(i)$, then $x_{i}=\lambda_{i} e_{u(i)}+\mu_{i} e_{w(i)}$ where $\lambda_{i}, \mu_{i} \neq 0$.

(iii) If $i>m$, then $x_{i}=e_{u(i)}$ or $x_{i}=e_{w(i)}$.

Then $\left\{x_{1}, \ldots, x_{n}\right\}$ is also a basis for $E$. The flag $V_{\bullet} \in \mathrm{F} \ell(E)$ given by $V_{i}=$ $\left\langle x_{1}, \ldots, x_{i}\right\rangle$ belongs to the Schubert variety $\Omega_{u}\left(F_{\bullet}\right)$ where $F_{\bullet}$ is defined by $F_{i}=$ $\left\langle e_{n+1-i}, \ldots, e_{n}\right\rangle$. Furthermore, this flag $V$. does not depend on the choices made in (iii).

Proof. Suppose at first that $x_{i}=e_{u(i)}$ for all $i>m$. In this case we have $F_{n+1-u(i)}=F_{n-u(i)} \oplus \mathbb{C} x_{i}$ for all $i$, so $\left\{x_{1}, \ldots, x_{n}\right\}$ is a basis and $V_{\bullet} \in \Omega_{u}\left(F_{\bullet}\right)$. It suffices to show that $V_{i-1} \oplus \mathbb{C} e_{u(i)}=V_{i-1} \oplus \mathbb{C} e_{w(i)}$ for each $i>m$. In case $u(i) \neq w(i)$ we let $b$ and $c$ be sequences satisfying Definition 1, such that for some $p$ we have $c_{1}=\cdots=c_{p}=i$ and $c_{j} \neq i$ for $j>p$. Then the values $w\left(b_{1}\right), w\left(b_{2}\right), \ldots, w\left(b_{p}\right), w(i)$ agree with $u(i), u\left(b_{1}\right), \ldots, u\left(b_{p-1}\right), u\left(b_{p}\right)$, in the indicated order. This implies that $\left\langle x_{b_{1}}, \ldots, x_{b_{p}}\right\rangle$ is a subspace of $\left\langle e_{u\left(b_{1}\right)}, \ldots, e_{u\left(b_{p}\right)}, e_{u(i)}\right\rangle$. Furthermore, (ii) implies that neither $e_{u(i)}$ nor $e_{w(i)}$ is contained in this subspace, so $\left\langle x_{b_{1}}, \ldots, x_{b_{p}}, e_{u(i)}\right\rangle=$ $\left\langle x_{b_{1}}, \ldots, x_{b_{p}}, e_{w(i)}\right\rangle=\left\langle e_{u\left(b_{1}\right)}, \ldots, e_{u\left(b_{p}\right)}, e_{u(i)}\right\rangle$. The required identity of subspaces follows from this.

We also need the following characterization of Pieri sequences, which is equivalent to parts (i) and (ii) of [7, Lemma 5.2].

Lemma 5. A sequence of non-negative integers $d=\left(d_{1}, \ldots, d_{k}\right)$ is a Pieri sequence with maximum at position $j$ if and only if the inequality

$$
d_{j}+\sum_{i=1}^{k-1} d_{i} d_{i+1}-\sum_{i=1}^{k} d_{i}^{2} \geq 0
$$

is satisfied. In this case the inequality is satisfied with equality.

Proof (Fomin). The inequality can be rewritten as $2 d_{j} \geq \sum_{i=0}^{k}\left(d_{i}-d_{i+1}\right)^{2}$, and the right-hand side of this is estimated from below by $\sum\left|d_{i}-d_{i+1}\right| \geq 2 d_{j}$.

\section{Geometric tools}

In this section we will give some tools for handling curves in flag varieties. It is convenient to extend the notation for partial flag varieties to allow weakly increasing sequences of dimensions. If $b=\left(b_{1} \leq b_{2} \leq \cdots \leq b_{k}\right)$ is a weakly increasing sequence with $b_{1} \geq 0$ and $b_{k} \leq n$, we let $\mathrm{F} \ell(b ; E)$ be the variety of partial flags $K_{1} \subset K_{2} \subset \cdots \subset K_{k} \subset E$ such that $\operatorname{dim} K_{i}=b_{i}$ for all $i$. The Schubert varieties in $\mathrm{F} \ell(b ; E)$ are indexed by the set $S_{n}(b)$ of permutations whose descent positions are contained in $\left\{b_{1}, \ldots, b_{k}\right\}$.

Let $b$ be a weakly increasing sequence such that $b_{i} \leq a_{i}$ for each $i$. Given a Schubert variety $\Omega_{w}^{(a)}\left(F_{\bullet}\right) \subset \mathrm{F} \ell(a ; E)$, we will need a description of the set of points $K_{\bullet} \in \mathrm{F} \ell(b ; E)$ such that for some $V_{\bullet} \in \Omega_{w}^{(a)}\left(F_{\bullet}\right)$ we have $K_{i} \subset V_{i}$ for all $i$.

We construct a permutation $\bar{w} \in S_{n}(b)$ from $w$ as follows. Set $w^{(0)}=w$. Then for each $1 \leq i \leq k$ we let $w^{(i)}$ be the permutation obtained from $w^{(i-1)}$ by rearranging the elements $w^{(i-1)}\left(b_{i}+1\right), \ldots, w^{(i-1)}\left(a_{i+1}\right)$ in increasing order. Finally we set 
$\bar{w}=w^{(k)}$. For example, if $n=6, a=(2,5), b=(1,2)$, and $w=263451$, then $w^{(1)}=234561$ and $\bar{w}=231456$. The following result is proved in 3].

Lemma 6. The set $\left\{K_{\bullet} \in \mathrm{F} \ell(b ; E) \mid \exists V_{\bullet} \in \Omega_{w}^{(a)}\left(F_{\bullet}\right): K_{i} \subset V_{i} \forall i\right\}$ is equal to the Schubert variety $\Omega_{\bar{w}}^{(b)}\left(F_{\bullet}\right)$ in $\mathrm{F} \ell(b ; E)$.

Our notation is related to Pieri sequences as follows.

Lemma 7. Let $d$ be a Pieri sequence and set $b=a-d=\left(a_{1}-d_{1}, \ldots, a_{k}-d_{k}\right)$. Let $u \in S_{n}(a)$. Then $\ell\left(u \gamma_{d}\right)=\ell(u)-\ell\left(\gamma_{d}\right)$ if and only if $\ell(\bar{u})=\ell(u)-\ell\left(\gamma_{d}\right)$ if and only if $u \gamma_{d}=\bar{u}$. In this case we have $u \gamma_{d} \in S_{n}(b)$.

Proof. With the notation of section 3.2 we have $\ell\left(\gamma_{d}\right)=\sum \ell\left(\tau_{i}\right)$. The lemma follows because $\ell\left(u^{(i-1)} \tau_{i}\right) \geq \ell\left(u^{(i-1)}\right)-\ell\left(\tau_{i}\right)$ and $\ell\left(u^{(i)}\right) \geq \ell\left(u^{(i-1)}\right)-\ell\left(\tau_{i}\right)$ for all $i$, with equality if and only if $u^{(i)}=u^{(i-1)} \tau_{i}$.

Now let $C \subset \mathrm{F} \ell(a ; E)$ be a rational curve of multidegree $d=\left(d_{1}, \ldots, d_{k}\right)$. For each $i$ we let $C_{i}=\rho_{i}(C) \subset \operatorname{Gr}\left(a_{i}, E\right)$ be the image of $C$ in the Grassmannian $\operatorname{Gr}\left(a_{i}, E\right)$ by the projection $\rho_{i}: \mathrm{F} \ell(a ; E) \rightarrow \operatorname{Gr}\left(a_{i}, E\right)$. This curve $C_{i}$ then has a kernel and a span [4]. The kernel is the largest subspace of $E$ contained in all the $a_{i}$-dimensional subspaces of $E$ corresponding to points of $C_{i}$. We let $b_{i}$ be the dimension of this kernel and denote the kernel itself by $K_{i}$. It follows from 4 Lemma 1] that $b_{i} \geq a_{i}-d_{i}$ for each $i$. The span of $C_{i}$ is the smallest subspace of $E$ containing all subspaces given by points of $C_{i}$. This span has dimension at most $a_{i}-d_{i}$.

The kernels $K_{i}$ form a partial flag $K_{\bullet} \in \mathrm{F} \ell(b ; E)$, called the kernel of $C$. Notice that $K_{i} \subset V_{i}$ for all points $V_{\bullet} \in C$. Lemma 6 therefore implies the following (cf. 3 Prop. 1]).

Proposition 1. Let $C \in \mathrm{F} \ell(a ; E)$ be a rational curve with kernel $K . \in \mathrm{F} \ell(b ; E)$. If $C \cap \Omega_{w}^{(a)}\left(F_{\bullet}\right) \neq \emptyset$, then $K_{\bullet} \in \Omega_{\bar{w}}^{(b)}\left(F_{\bullet}\right)$.

Lemma 8. Let $f: \mathbb{P}^{1} \rightarrow \operatorname{Gr}(m, E)$ be a curve of degree d such that the kernel $K$ of $f\left(\mathbb{P}^{1}\right)$ has dimension $m-d$. Then there are elements $x_{1}, \ldots, x_{d}, y_{1}, \ldots, y_{d} \in E$ such that $f(s: t)=K \oplus\left\langle s x_{1}+t y_{1}, \ldots, s x_{d}+t y_{d}\right\rangle$ for all $(s: t) \in \mathbb{P}^{1}$.

Proof. Any regular map $f: \mathbb{P}^{1} \rightarrow \operatorname{Gr}(m, E)$ can be written in the form $f(s: t)=$ $\left\langle f_{1}(s: t), \ldots, f_{m}(s: t)\right\rangle$ for regular maps $f_{i}: \mathbb{P}^{1} \rightarrow \mathbb{P}(E)$, and furthermore we have $\sum \operatorname{deg}\left(f_{i}\right)=\operatorname{deg}(f)=d$. (To see this, one uses that the pullback of the tautological subbundle on $\operatorname{Gr}(m ; E)$ splits as a sum of line bundles on $\mathbb{P}^{1}$.) At least $m-d$ of these maps must have degree zero, so we can assume that $f_{d+1}, \ldots, f_{m}$ are constant. Since $\left\langle f_{d+1}, \ldots, f_{m}\right\rangle$ is contained in $K$ and these spaces have the same dimension, we conclude that $K=\left\langle f_{d+1}, \ldots, f_{m}\right\rangle$. This implies that none of the functions $f_{1}, \ldots, f_{d}$ are constant, so they must all have degree one. Thus we can write $f_{i}(s: t)=s x_{i}+t y_{i}$ for some $x_{i}, y_{i} \in E$ for $1 \leq i \leq d$.

Given a morphism $f: \mathbb{P}^{1} \rightarrow \mathrm{F} \ell(a ; E)$, we let $f_{i}: \mathbb{P}^{1} \rightarrow \operatorname{Gr}\left(a_{i}, E\right)$ denote the composition of $f$ with the $i$ th projection $\rho_{i}: \mathrm{F} \ell(a ; E) \rightarrow \operatorname{Gr}\left(a_{i}, E\right)$.

Lemma 9. Let $a=\left(a_{1}<a_{2}<a_{3}\right)$ be a sequence of integers, $0<a_{i}<n$, and let $f=\left(f_{1}, f_{2}, f_{3}\right): \mathbb{P}^{1} \rightarrow \mathrm{F} \ell(a ; E)$ be a regular map of multidegree $(d, d+1, d)$ for some integer $d \geq 0$. Suppose that the kernel $K_{\bullet} \in \mathrm{F} \ell(b ; E)$ of $f\left(\mathbb{P}^{1}\right)$ has dimensions given by $b=\left(a_{1}-d, a_{2}-d-1, a_{3}-d\right)$. Suppose also there are linearly independent 
elements $x_{1}, \ldots, x_{d+1}, y_{1}, \ldots, y_{d+1} \in E$ such that

(1) $f_{1}(s: t)=K_{1} \oplus\left\langle s x_{1}+t y_{1}, \ldots, s x_{d}+t y_{d}\right\rangle$ for all $(s: t) \in \mathbb{P}^{1}$,

(2) $f_{2}(1: 0)=K_{2} \oplus\left\langle x_{1}, \ldots, x_{d+1}\right\rangle$,

(3) $f_{2}(0: 1)=K_{2} \oplus\left\langle y_{1}, \ldots, y_{d+1}\right\rangle$,

(4) $K_{2} \cap\left\langle x_{1}, \ldots, x_{d+1}, y_{1}, \ldots, y_{d+1}\right\rangle=0$,

(5) $K_{3} \cap\left\langle x_{1}, \ldots, x_{d}, y_{1}, \ldots, y_{d}\right\rangle=0$, and

(6) $x_{d+1}, y_{d+1} \in K_{3}$.

Then there exists a unique $\lambda \in \mathbb{C}^{*}$ such that

$$
f_{2}(s: t)=K_{2} \oplus\left\langle s x_{1}+t y_{1}, \ldots, s x_{d}+t y_{d}, s x_{d+1}+t \lambda y_{d+1}\right\rangle .
$$

Proof. By Lemma 8 we can find elements $x_{1}^{\prime}, \ldots, x_{d+1}^{\prime}, y_{1}^{\prime}, \ldots, y_{d+1}^{\prime} \in E$ such that $f_{2}(s: t)=K_{2} \oplus\left\langle s x_{1}^{\prime}+t y_{1}^{\prime}, \ldots, s x_{d+1}^{\prime}+t y_{d+1}^{\prime}\right\rangle$. Using (2) we can write $x_{i}=$ $z_{i}+\sum_{j=1}^{d+1} \alpha_{i j} x_{j}^{\prime}$ for each $i$ where $z_{i} \in K_{2}$ and $\left(\alpha_{i j}\right)$ is an invertible matrix. Replacing $x_{i}^{\prime}$ with $x_{i}$ and $y_{i}^{\prime}$ with $\sum_{j} \alpha_{i j} y_{j}^{\prime}$ we may assume that $x_{i}^{\prime}=x_{i}$ for each $i$.

Now if $i \leq d$ we have $x_{i}+y_{i} \in f_{1}(1: 1) \subset f_{2}(1: 1)$ by (1), so we may write $x_{i}+y_{i}=z_{i}^{\prime}+\sum_{j=1}^{d+1} \beta_{i j}\left(x_{j}+y_{j}^{\prime}\right)=\sum \beta_{i j} x_{j}+\left(z_{i}^{\prime}+\sum \beta_{i j} y_{j}^{\prime}\right)$ where $z_{i}^{\prime} \in K_{2}$ and $\beta_{i j} \in \mathbb{C}$. Since the last term of this belongs to $K_{2} \oplus\left\langle y_{1}, \ldots, y_{d+1}\right\rangle$, it follows from (4) that $\beta_{i j}$ is equal to one if $i=j$ and zero otherwise, so we conclude that $x_{i}+y_{i}=z_{i}^{\prime}+\left(x_{i}+y_{i}^{\prime}\right)$. Thus we have $y_{i}=y_{i}^{\prime}+z_{i}^{\prime}$, so we may replace $y_{i}^{\prime}$ with $y_{i}$ for $1 \leq i \leq d$.

Finally, since $y_{d+1}^{\prime} \in f_{2}(0: 1)$, we can write $y_{d+1}^{\prime}=z^{\prime \prime}+\sum_{j=1}^{d+1} \lambda_{j} y_{j}$ by (3) where $z^{\prime \prime} \in K_{2}, \lambda_{j} \in \mathbb{C}$. Replacing $y_{d+1}^{\prime}$ with $y_{d+1}^{\prime}-z^{\prime \prime}$ we may assume that $z^{\prime \prime}=0$. Now (1) and (5) imply that $f_{3}(1: 1)=K_{3} \oplus\left\langle x_{1}+y_{1}, \ldots, x_{d}+y_{d}\right\rangle$. Since $x_{d+1}+y_{d+1}^{\prime} \in$ $f_{2}(1: 1) \subset f_{3}(1: 1)$, we conclude by (5) and (6) that $\lambda_{j}=0$ for $j \leq d$. So we have $y_{d+1}^{\prime}=\lambda_{d+1} y_{d+1}$, as required.

\section{Proof of the quantum Pieri formula}

In this section we finally prove Ciocan-Fontanine's quantum Pieri formula [7]. For convenience we will prove the following equivalent version of Theorem 1.

Theorem 1'. Let $\alpha=s_{r} s_{r+1} \cdots s_{a_{j}}$ and $u, w \in S_{n}(a)$ be permutations, and let $d=\left(d_{1}, \ldots, d_{k}\right)$ be a multidegree, such that $\ell(u)+\ell(w)+\ell(\alpha)=\operatorname{dim} \mathrm{F} \ell(a ; E)+$ $\sum\left(a_{i+1}-a_{i-1}\right) d_{i}$. The Gromov-Witten invariant $\left\langle\Omega_{u}^{(a)}, \Omega_{w}^{(a)}, \Omega_{\alpha}^{(a)}\right\rangle_{d}$ on $\mathrm{F} \ell(a ; E)$ is non-zero only if $d$ is a Pieri sequence with maximum at position $j$. In this case we have

$$
\left\langle\Omega_{u}^{(a)}, \Omega_{w}^{(a)}, \Omega_{\alpha}^{(a)}\right\rangle_{d}=\int_{\mathrm{F} \ell(b ; E)} \Omega_{\bar{u}}^{(b)} \cdot \Omega_{\bar{w}}^{(b)} \cdot \Omega_{\bar{\alpha}}^{(b)}
$$

where $b=a-d=\left(a_{1}-d_{1}, \ldots, a_{k}-d_{k}\right)$.

It will be clear from the proof that, if the right hand side of the identity is nonzero, then $\ell(\bar{u})=\ell(u)-\sum\left(a_{i+1}-a_{i}\right) d_{i}=\ell(u)-\ell\left(\gamma_{d}\right)$, so $\bar{u}=u \gamma_{d}$ by Lemma 7 and similarly for $w$. Therefore the equivalence with Theorem 1 is a matter of dualizing the permutation $w$. Notice also that the right hand side can only be equal to zero or one by the classical Pieri formula (11).

Proof. We first show that if the Gromov-Witten invariant $\left\langle\Omega_{u}^{(a)}, \Omega_{w}^{(a)}, \Omega_{\alpha}^{(a)}\right\rangle_{d}$ is nonzero, then $d$ is a Pieri sequence with maximum at position $j$, and the triple intersection on $\mathrm{F} \ell(b ; E)$ is non-zero as well. Throughout this proof, $F_{\bullet}, G_{\bullet}$, and $H_{\bullet}$ will denote full flags of $E$ in general position. 
Let $C \subset \mathrm{F} \ell(a ; E)$ be a rational curve of multidegree $d$ which meets each of the Schubert varieties $\Omega_{u}^{(a)}\left(F_{\bullet}\right), \Omega_{w}^{(a)}\left(G_{\bullet}\right)$, and $\Omega_{\alpha}^{(a)}\left(H_{\bullet}\right)$. Let $K_{\bullet} \in \mathrm{F} \ell(b ; E)$ be the kernel of $C$ and set $e_{i}=a_{i}-b_{i}$ for $1 \leq i \leq k$. Then [4, Lemma 1] shows that $e_{i} \leq d_{i}$ for all $i$, and by Proposition 1 we have $K_{\bullet} \in \Omega_{\bar{u}}^{(b)}\left(F_{\bullet}\right) \cap \Omega_{\bar{w}}^{(b)}\left(G_{\bullet}\right) \cap \Omega_{\bar{\alpha}}^{(b)}\left(H_{\bullet}\right)$. In particular $\ell(\bar{u})+\ell(\bar{w})+\ell(\bar{\alpha}) \leq \operatorname{dim} \mathrm{F} \ell(b ; E)$. By the definition of $\bar{w}$ we get $\ell(\bar{\alpha}) \geq$ $\ell(\alpha)-e_{j}, \ell(\bar{u}) \geq \ell(u)-\sum_{i=1}^{k}\left(a_{i+1}-a_{i}\right) e_{i}$, and $\ell(\bar{w}) \geq \ell(w)-\sum_{i=1}^{k}\left(a_{i+1}-a_{i}\right) e_{i}$. Thus we obtain

$\operatorname{dim} \mathrm{F} \ell(b ; E) \geq \ell(\bar{u})+\ell(\bar{w})+\ell(\bar{\alpha}) \geq \operatorname{dim} \mathrm{F} \ell(a ; E)-e_{j}+\sum_{i=1}^{k}\left(2 a_{i}-a_{i-1}-a_{i+1}\right) e_{i}$.

Since $\operatorname{dim} \mathrm{F} \ell(b ; E)-\operatorname{dim} \mathrm{F} \ell(a ; E)=\sum\left(2 a_{i}-a_{i-1}-a_{i+1}\right) e_{i}+\sum\left(e_{i} e_{i+1}-e_{i}^{2}\right)$, this implies that

$$
e_{j}+\sum e_{i} e_{i+1}-\sum e_{i}^{2} \geq 0
$$

Lemma 5 therefore shows that $e$ is a Pieri sequence with maximum at position $j$, and that all the inequalities above must be satisfied with equality. In particular we have $d=e$. Furthermore, since $\ell(\bar{u})+\ell(\bar{w})+\ell(\bar{\alpha})=\operatorname{dim} \mathrm{F} \ell(b ; E)$, we must have $\int \Omega_{\bar{u}}^{(b)} \cdot \Omega_{\bar{w}}^{(b)} \cdot \Omega_{\bar{\alpha}}^{(b)}=1$, as required.

On the other hand, if $d$ is a Pieri sequence with maximum at position $j$, and if the triple intersection on $\mathrm{F} \ell(b ; E)$ is non-zero, then the same dimension count shows that $\ell(\bar{u})=\ell(u)-\ell\left(\gamma_{d}\right)$ and $\ell(\bar{w})=\ell(w)-\ell\left(\gamma_{d}\right)$, so $\bar{u}=u \gamma_{d}$ and $\bar{w}=w \gamma_{d}$ by Lemma 7 Since $\ell(\bar{\alpha})=\ell(\alpha)-d_{j}$, we deduce that $\bar{\alpha}=s_{r} s_{r+1} \cdots s_{b_{j}}$. We will continue by showing that the Gromov-Witten invariant $\left\langle\Omega_{u}^{(a)}, \Omega_{w}^{(a)}, \Omega_{\alpha}^{(a)}\right\rangle_{d}$ is non-zero by explicitly constructing a rational curve $C \subset \mathrm{F} \ell(a ; E)$ of multidegree $d$, which meets each of the Schubert varieties $\Omega_{u}^{(a)}\left(F_{\bullet}\right), \Omega_{w}^{(a)}\left(G_{\bullet}\right)$, and $\Omega_{\alpha}^{(a)}\left(H_{\bullet}\right)$.

As in $\$ 3.2$ we set $h_{p}=\min \left\{i: d_{i}=p\right\}$ and $l_{p}=\max \left\{i: d_{i}=p\right\}$ for $1 \leq p \leq d_{j}$, so that $\gamma_{d}\left(b_{l_{p}+1}\right)=a_{h_{p}}$, while $\gamma_{d}(i)=i+p$ if $b_{h_{p}}<i \leq b_{h_{p+1}}$ or if $b_{l_{p+1}+1}<i<b_{l_{p}+1}$. We also set $E_{i}=F_{n+1-i} \cap G_{i}$ for $1 \leq i \leq n$. Since the flags are general, it follows that these spaces have dimension one, and $E=E_{1} \oplus \cdots \oplus E_{n}$.

Let $\widetilde{w}=w_{0} \bar{w} w_{b}$ be the dual permutation of $\bar{w} \in S_{n}(b)$. Then $\bar{u} \stackrel{\bar{\alpha}}{\longrightarrow} \widetilde{w}$. For each $1 \leq i \leq n$ we define a space $L_{i} \subset E$ as follows. If $\bar{u}(i)=\widetilde{w}(i)$ or if $i>b_{j}$, we set $L_{i}=E_{\bar{u}(i)}$. Let $B$ be the direct sum of the spaces $L_{i}$ for which $i \leq b_{j}$ and $\bar{u}(i)=\widetilde{w}(i)$. Notice that $\operatorname{dim} B=r-1$. When $i \leq b_{j}$ and $\bar{u}(i) \neq \widetilde{w}(i)$ we then let $L_{i}$ be the unique one-dimensional subspace of $E_{\bar{u}(i)} \oplus E_{\widetilde{w}(i)}$ such that $\left(B \oplus L_{i}\right) \cap H_{n-r} \neq 0$. This is well defined, since $B \oplus E_{\bar{u}(i)} \oplus E_{\widetilde{w}(i)}$ has dimension $r+1$ and since the flags are general; and furthermore we have $L_{i} \neq E_{\bar{u}(i)}$ and $L_{i} \neq E_{\widetilde{w}(i)}$.

It follows from Lemma 4 that the spaces $L_{i}$ are linearly independent and that the partial flag $K_{\bullet} \in \mathrm{F} \ell(b ; E)$ defined by $K_{i}=L_{1} \oplus \cdots \oplus L_{b_{i}}$ belongs to the Schubert variety $\Omega_{\bar{u}}^{(b)}\left(F_{\bullet}\right)$. We furthermore get the same partial flag $K_{\bullet}$ if we take $L_{i}=E_{\widetilde{w}(i)}$

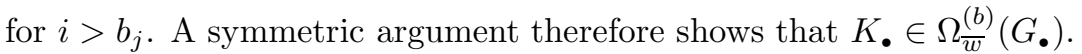

Finally, since $\left(B \oplus L_{i}\right) \cap H_{n-r} \neq 0$ for $\ell(\bar{\alpha})$ different indices $i \leq b_{j}$, we obtain $\operatorname{dim}\left(K_{j} \cap H_{n-r}\right)=\ell(\bar{\alpha})$, which means that $K_{\bullet} \in \Omega_{\bar{\alpha}}^{(b)}\left(H_{\bullet}\right)$. By the classical Pieri formula we therefore conclude that

$$
\Omega_{\bar{u}}^{(b)}\left(F_{\bullet}\right) \cap \Omega_{\bar{w}}^{(b)}\left(G_{\bullet}\right) \cap \Omega_{\bar{\alpha}}^{(b)}\left(H_{\bullet}\right)=\left\{K_{\bullet}\right\} .
$$


Notice that this implies that $K$. must be the kernel of any rational curve of multidegree $d$ in $\mathrm{F} \ell(a ; E)$ which passes through $\Omega_{u}^{(a)}\left(F_{\bullet}\right), \Omega_{w}^{(a)}\left(G_{\bullet}\right)$, and $\Omega_{\alpha}^{(a)}\left(H_{\bullet}\right)$.

Now for each $1 \leq p \leq m=d_{j}$ choose $x_{p} \in E_{u\left(a_{h_{p}}\right)}$ and $y_{p} \in E_{w_{0} w\left(a_{h_{p}}\right)}$ such that $\left(B \oplus\left\langle x_{p}+y_{p}\right\rangle\right) \cap H_{n-r} \neq 0$. Notice that since $\gamma_{d}\left(b_{l_{p}+1}\right)=a_{h_{p}}$, we have $u\left(a_{h_{p}}\right)=\bar{u}\left(b_{l_{p}+1}\right)$ and $w_{0} w\left(a_{h_{p}}\right)=\widetilde{w}\left(b_{l_{p}}+1\right)$. By Lemma 4 this implies that $B \cap\left(E_{u\left(a_{h_{p}}\right)} \oplus E_{w_{0} w\left(a_{h_{p}}\right)}\right)=0$, so $x_{p}$ and $y_{p}$ can be found. Since the integers $b_{l_{i}+1}$ and $b_{l_{i}}+1$ are all different for $1 \leq i \leq m$, the same lemma furthermore implies that $x_{1}, \ldots, x_{m}, y_{1}, \ldots, y_{m}$ are linearly independent and that $K_{l_{p}} \cap\left\langle x_{1}, \ldots, x_{p}, y_{1}, \ldots, y_{p}\right\rangle=0$ for all $p$.

Let $f: \mathbb{P}^{1} \rightarrow \mathrm{F} \ell(a ; E)$ be the morphism which maps a point $(s: t) \in \mathbb{P}^{1}$ to the partial flag $V_{\bullet} \in \mathrm{F} \ell(a ; E)$ given by $V_{i}=K_{i} \oplus\left\langle s x_{1}+t y_{1}, \ldots, s x_{d_{i}}+t y_{d_{i}}\right\rangle$. Since $x_{p}, y_{p} \in K_{l_{p}+1}$, it follows that $V_{i} \subset V_{i+1}$ for all $i$, so $f$ is well defined. Its image $C=f\left(\mathbb{P}^{1}\right) \subset \mathrm{F} \ell(a ; E)$ is a rational curve of multidegree $d$.

Notice that $F_{n+1-\bar{u}(i)}=F_{n-\bar{u}(i)} \oplus L_{i}$. If we set $L_{p}^{\prime}=L_{\gamma_{d}^{-1}(p)}$ for $1 \leq p \leq n$, then $F_{n+1-u(p)}=F_{n-u(p)} \oplus L_{p}^{\prime}$ and the space of dimension $a_{i}$ in the partial flag $f(1: 0)$ is equal to $L_{1}^{\prime} \oplus \cdots \oplus L_{a_{i}}^{\prime}$. This shows that $f(1: 0) \in \Omega_{u}^{(a)}\left(F_{\text {. }}\right)$. A symmetric argument shows that $f(0: 1) \in \Omega_{w}^{(a)}\left(G_{\bullet}\right)$. Finally, since $\operatorname{dim}\left(K_{j} \cap H_{n-r}\right)=\ell(\alpha)-d_{j}$ and since $\left(B \oplus\left\langle x_{i}+y_{i}\right\rangle\right) \cap H_{n-r} \neq 0$ for $1 \leq i \leq d_{j}$ we conclude that $f(1: 1) \in \Omega_{\alpha}^{(a)}\left(H_{\bullet}\right)$. This proves that $\left\langle\Omega_{u}^{(a)}, \Omega_{w}^{(a)}, \Omega_{\alpha}^{(a)}\right\rangle_{d} \neq 0$.

It remains to be shown that $C$ is the only curve which contributes to this GromovWitten invariant. Let $f^{\prime}: \mathbb{P}^{1} \rightarrow \mathrm{F} \ell(a ; E)$ be any rational curve of multidegree $d$ such that $f^{\prime}(1: 0) \in \Omega_{u}^{(a)}\left(F_{\bullet}\right), f^{\prime}(0: 1) \in \Omega_{w}^{(a)}\left(G_{\bullet}\right)$, and $f^{\prime}(1: 1) \in \Omega_{\alpha}^{(a)}\left(H_{\bullet}\right)$. Then the kernel of $f^{\prime}\left(\mathbb{P}^{1}\right)$ must be $K_{\text {. }}$. We will show that $f^{\prime}$ is identical to the map $f$ constructed above.

Set $f_{i}^{\prime}=\rho_{i} \circ f^{\prime}$ and $f_{i}=\rho_{i} \circ f: \mathbb{P}^{1} \rightarrow \operatorname{Gr}\left(a_{i}, E\right)$. We will prove that $f_{i}^{\prime}=f_{i}$ by induction on $i$, the case $i=0$ being clear. Assume that $i>0$ and that $f_{i-1}^{\prime}=f_{i-1}$. If $i \notin\left\{h_{1}, \ldots, h_{m}\right\}$, then this follows because

$$
f_{i}^{\prime}(s: t) \supset f_{i-1}^{\prime}(s: t)+K_{i}=f_{i-1}(s: t)+K_{i}=f_{i}(s: t)
$$

by the definition of $f$.

So suppose $i=h_{p}$ for some $p$. Then we know that $f_{h_{p}-1}^{\prime}=f_{h_{p}-1}$. Since $f_{l_{p}+1}^{\prime}(s: t)$ must contain $f_{h_{p}-1}(s: t)+K_{l_{p}+1}=f_{l_{p}+1}(s: t)$, we furthermore deduce that $f_{l_{p}+1}^{\prime}=f_{l_{p}+1}$. In particular we see that the span of $f_{l_{p}+1}^{\prime}\left(\mathbb{P}^{1}\right)$ is the space $W=K_{l_{p}+1} \oplus\left\langle x_{1}, \ldots, x_{p-1}, y_{1}, \ldots, y_{p-1}\right\rangle$.

Notice that since $\ell\left(u \gamma_{d}\right)=\ell(u)-\ell\left(\gamma_{d}\right)$ we have $u\left(a_{h_{\tau}}\right)>u\left(a_{h_{p}}\right)$ for $\tau<p$ (see the remarks after Theorem 11). Similarly we have $w_{0} w\left(a_{h_{\tau}}\right)<w_{0} w\left(a_{h_{p}}\right)=$ $\widetilde{w}\left(b_{l_{p}}+1\right) \leq \bar{u}\left(b_{l_{p}}+1\right) \leq \bar{u}\left(b_{l_{p}+1}\right)=u\left(a_{h_{p}}\right)$.

We claim that the intersection of $W$ with $F_{n+1-u\left(a_{i}\right)}$ is contained in $K_{i} \oplus$ $\left\langle x_{1}, \ldots, x_{p-1}\right\rangle \oplus E_{u\left(a_{i}\right)}$. To see this, notice at first that $x_{1}, \ldots, x_{p-1} \in F_{n-u\left(a_{i}\right)}$ and $y_{1}, \ldots, y_{p-1} \in G_{u\left(a_{i}\right)}$ by the above inequalities. We will show that for any $\tau \leq b_{l_{p}+1}$ we have $L_{\tau} \subset F_{n-u\left(a_{i}\right)}$ or $L_{\tau} \subset G_{u\left(a_{i}\right)}$. Again using that $\ell\left(u \gamma_{d}\right)=\ell(u)-\ell\left(\gamma_{d}\right)$, it follows that $u\left(a_{i}\right)=\bar{u}\left(b_{l_{p}+1}\right) \geq \bar{u}(\tau)$ for all $b_{i}<\tau \leq b_{l_{p}+1}$. If $b_{j}<\tau \leq b_{l_{p}+1}$, we therefore get $L_{\tau}=E_{\bar{u}(\tau)} \subset G_{u\left(a_{i}\right)}$. If $\tau \leq b_{j}$, then $\bar{u}(\tau) \leq \widetilde{w}(\tau)$. So if $\bar{u}(\tau)>u\left(a_{i}\right)$, then $L_{\tau} \subset F_{n-u\left(a_{i}\right)}$. Finally, if $\tau \leq b_{j}$ and $\bar{u}(\tau) \leq u\left(a_{i}\right)$, then it follows from Lemma 3 that $\widetilde{w}(\tau) \leq u\left(a_{i}\right)$, so $L_{\tau} \subset G_{u\left(a_{i}\right)}$. Since in particular $L_{\tau} \subset G_{u\left(a_{i}\right)}$ for all $b_{i}<\tau \leq b_{l_{p}+1}$, it follows that $W \cap F_{n-u\left(a_{i}\right)} \subset K_{i} \oplus\left\langle x_{1}, \ldots, x_{p-1}\right\rangle$. Our claim follows from this since $E_{u\left(a_{i}\right)} \subset W$. 
Since $F_{n+1-u(\tau)}=F_{n-u(\tau)} \oplus L_{\tau}^{\prime}$ for all $\tau$, it follows that the intersection of $K_{i} \oplus\left\langle x_{1}, \ldots, x_{p-1}\right\rangle=L_{1}^{\prime} \oplus \cdots \oplus L_{a_{i}-1}^{\prime}$ with $F_{n+1-u\left(a_{i}\right)}$ has dimension \# $\left\{\tau \leq a_{i} \mid\right.$ $\left.u(\tau) \geq u\left(a_{i}\right)\right\}-1$. Therefore we can write $f_{i}^{\prime}(1: 0)=K_{i} \oplus\left\langle x_{1}, \ldots, x_{p-1}, x_{p}^{\prime}\right\rangle$ where $x_{p}^{\prime} \in F_{n+1-u\left(a_{i}\right)}$. Since $x_{p}^{\prime}$ must also be contained in $W$, it follows from the claim that we can take $x_{p}^{\prime} \in E_{u\left(a_{i}\right)}$. A symmetric argument shows that

$$
f_{i}^{\prime}(0: 1)=K_{i} \oplus\left\langle y_{1}, \ldots, y_{p-1}, y_{p}^{\prime}\right\rangle
$$

where $y_{p}^{\prime} \in E_{w_{0} w\left(a_{i}\right)}$. Applying Lemma 9 to the map

$$
\left(f_{h_{p}-1}, f_{h_{p}}, f_{l_{p}+1}\right): \mathbb{P}^{1} \rightarrow \mathrm{F} \ell\left(a_{h_{p}-1}, a_{h_{p}}, a_{l_{p}+1} ; E\right),
$$

we then conclude that

$$
f_{i}^{\prime}(s: t)=K_{i} \oplus\left\langle s x_{1}+t y_{1}, \ldots, s x_{p-1}+t y_{p-1}, s x_{p}^{\prime}+t \lambda y_{p}^{\prime}\right\rangle
$$

for some $\lambda \in \mathbb{C}^{*}$.

Finally, notice that we can write $f_{j}^{\prime}(1: 1)=B \oplus\left\langle x_{p}^{\prime}+\lambda y_{p}^{\prime}\right\rangle \oplus M$ for some subspace $M \subset E$ of dimension $\ell(\alpha)-1$. Since the dimension of $f_{j}^{\prime}(1: 1) \cap H_{n-r}$ is at least $\ell(\alpha)$, it follows that $\left(B \oplus\left\langle x_{p}^{\prime}+\lambda y_{p}^{\prime}\right\rangle\right) \cap H_{n-r} \neq 0$, so $\mathbb{C}\left(x_{p}^{\prime}+\lambda y_{p}^{\prime}\right)=\mathbb{C}\left(x_{p}+y_{p}\right)$. This shows that $f_{i}^{\prime}=f_{i}$ and finishes the proof.

\section{REFERENCES}

[1] A. Astashkevich and V. Sadov, Quantum cohomology of partial flag manifolds $F_{n_{1} \cdots n_{k}}$, Comm. Math. Phys. 170 (1995), 503-528. MR96g:58027

[2] A. Bertram, Quantum Schubert calculus, Adv. Math. 128 (1997), 289-305. MR98j:14067

[3] A. S. Buch, A direct proof of the quantum version of Monk's formula, Proc. Amer. Math. Soc. 131 (2003), 2037-2042. MR2004e:14081

[4] _ Quantum cohomology of Grassmannians, Compositio Math. 137 (2003), 227-235. MR.2004c:14105

[5] A. S. Buch, A. Kresch, H. Tamvakis, and A. Yong, Schubert polynomials and quiver formulas, Duke Math. J. 122 (2004), no. 1, 125-143.

[6] I. Ciocan-Fontanine, Quantum cohomology of flag varieties, Internat. Math. Res. Notices (1995), 263-277. MR96h:14071

[7] _ On quantum cohomology rings of partial flag varieties, Duke Math. J. 98 (1999), 485-524. MR2000d:14058

[8] S. Fomin, S. Gelfand, and A. Postnikov, Quantum Schubert polynomials, J. Amer. Math. Soc. 10 (1997), 565-596. MR 98d:14063

[9] W. Fulton and R. Pandharipande, Notes on stable maps and quantum cohomology, Algebraic geometry - Santa Cruz 1995, Proc. Sympos. Pure Math., vol. 62, 1997, pp. 45-96. MR.98m:14025

[10] A. Givental and B. Kim, Quantum cohomology of flag manifolds and Toda lattices, Comm. Math. Phys. 168 (1995), 609-641. MR96c:58027

[11] B. Kim, Quantum cohomology of partial flag manifolds and a residue formula for their intersection pairings, Internat. Math. Res. Notices (1995), 1-15. MR96c:58028

[12] On equivariant quantum cohomology, Internat. Math. Res. Notices (1996), 841-851. MR 98h:14013

[13] Quantum cohomology of flag manifolds $G / B$ and quantum Toda lattices, Ann. of Math. (2) 149 (1999),129-148. MR2001c:14081

[14] M. Kontsevich and Yu. Manin, Gromov-Witten classes, quantum cohomology, and enumerative geometry, Mirror symmetry, II, AMS/IP Stud. Adv. Math., vol. 1, 1997, pp. 607-653. MR $97 \mathrm{~d}: 00024$

[15] A. Lascoux and M.-P. Schützenberger, Polynômes de Schubert, C. R. Acad. Sci. Paris Sér. I Math. 294 (1982), 447-450. MR83e:14039

[16] I. G. Macdonald, Notes on Schubert polynomials, Laboratoire de Combinatoire et d’Informatique Mathématique, Université du Québec à Montréal, 1991. 
[17] A. Postnikov, On a quantum version of Pieri's formula, Advances in geometry, Progr. Math., vol. 172, 1999, pp. 371-383. MF99m:14096

[18] Y. Ruan and G. Tian, A mathematical theory of quantum cohomology, Math. Res. Lett. 1 (1994), 269-278. MR95b:58025

[19] B. Siebert and G. Tian, On quantum cohomology rings of Fano manifolds and a formula of Vafa and Intriligator, Asian J. Math. 1 (1997), 679-695. MF99d:14060

[20] F. Sottile, Pieri's formula for flag manifolds and Schubert polynomials, Ann. Inst. Fourier (Grenoble) 46 (1996), 89-110. MR97g:14035

[21] E. Witten, The Verlinde algebra and the cohomology of the Grassmannian, Geometry, topology, \& physics, Conf. Proc. Lecture Notes Geom. Topology, IV, 1995, pp. 357-422. MR 98c:58016

Matematisk Institut, Aarhus Universitet, Ny Munkegade, 8000 Århus C, Denmark

E-mail address: abuch@imf.au.dk 